\title{
EFFECTS OF SYNTHETIC AND ORGANIC ACARICIDES ON HONEY BEE HEALTH: A REVIEW
}

\author{
Erik Tihelka \\ Hartpury College, Gloucestershire, United Kingdom \\ E-mail:erik.tihelka@hartpury.ac.uk
}

\begin{abstract}
Thehoney bee is a crucial pollinator of agricultural crops and also aneconomically important producer of commodities such as honey and beeswax that find diverse uses in the food industry, cosmetics and medicine. At present, the ectoparasitic mite Varroa destructor is viewed as the most damaging pest of the honey bee worldwide. Without treatment, colonies generally collapse within a few years. To keep the population of the Varroa mites low, beekeepers relay on the use of synthetic and organic acaricides, the most popular commercially available ones include amitraz, coumaphos, flumethrin, fluvalinate, formic acid, oxalic acid and thymol. These conventional acaricides are cheap and easy to apply, but prolonged use causes Varroa mites to rapidly develop resistance and bee products can become contaminated. Residues of acaricides are present in high concentrations throughout the hive and bees are exposed to them all year around. The present review summarises the current knowledge of the deleterious effects of conventional acaricides on honey bee health. Numerous commercially available acaricides and their active substances have been shown to have negative effects on honey bee brood development, queen and drone reproductive health, learning, longevity and colony strength. Acaricides do not only act alone, but also in synergic combinations to affect bee health. Since some drugs cause substantial weakening of bee colonies, they can make them more susceptible to other diseases such as nosematosis or to extreme climatic events. As wax combs are contaminated with high concentrations of acaricide residues and Varroa mites are chronically exposed to them, the parasite may develop resistance faster. In combination with other stressors, acaricides could be a contributing factor to colony collapses.
\end{abstract}

Key words: synthetic acaricides; varroacides; honey bee; Apis mellifera; synergy; organic beekeeping;

\section{Introduction}

The honey bee (Apis mellifera L. 1758) ranks among the most economically important pollinators in the world, providing key ecosystem services in both artificial and natural landscapes (1). It has been estimated that the worldwide value of pollination is about $€ 153$ billion annually (2), making honey bees key contributors to global economy. Also important is the role of honey bees

Received: 19 May 2017

Accepted for publication: 11 December 2017 in collecting and manufacturing products including honey, beeswax, propolis, royal jelly or bee venom that today find wide uses as foods, natural medicines and cosmetics. Beekeeping is the source of income for many around the world and a key poverty alleviation measure (3). However, there are a number of pests and parasites of the honey bee that require control. As of present, the Varroa mite (Varroa destructor) is considered to be the most damaging parasite of the honey bee worldwide (4). Without intervention most, but not all, colonies collapse within several years. Today, Varroa treatments consist primarily of chemotherapy (5). 
In Europe, the most frequently used chemicals to control $V$. destructor are synthetic compounds such as coumaphos (active ingredient in medicaments such as Check-Mite ${ }^{\mathrm{TM}}$ or Perizin ${ }^{\circledR}$ ), fluvalinate $\quad$ Apistan $^{\circledR}, \quad$ Gabon $\left.^{\circledR}\right)$, flumethrin $\left(\right.$ Bayvarol $^{\circledR}$ ) and amitraz $\left(\right.$ Apivar $^{\circledR}$, Varidol $\left.^{\circledR}\right)$ (6). In attempt to find more "natural" cures to varroasis, oxalic acid, formic acid $\left(\right.$ Formidol $\left.^{\mathbb{}}\right)$ and thymol $\left(\right.$ Thymovar $^{\circledR}$ ) have been introduced and are becoming increasingly popular among organic beekeepers (7). Organic acids and thymol occur naturally in low concentrations in honey, thereby their organic reputation. An overview of the most popular acaricidal chemicals used globally is given in Tab. 1. Today, many of the medicaments originally developed to control $V$. destructor are also used to control other widespread bee parasites such as Tropilaelaps mites or bee lice (Braula coeca) $(8,9)$. bee products (13). Residues of some acaricides have been shown to be very persistent and present a concern for human health (14). Equally importantly, the residues of acaricides can also have serious consequences for the health of the colony.

As of present, much research concentrated on the quantification of acaricide residues in hive products but relatively few studies addressed the impact of residues on honey bee health. This question is also highly controversial given the financial interest of a number of bee research organisations in manufacturing and selling bee medicaments. This review presents a compilation of the published effects of common commercially available synthetic and organic acaricides on honey bee health.

A review of the negative side effects of acaricides is listed separately for every active ingredient

Table 1: Examples of popular acaricides used across the world (10)

\begin{tabular}{|l|l|l|l|}
\hline Compound & Examples of Commercial Products & Residues & Resistance \\
\hline Amitraz & $\begin{array}{l}\text { Apivar } \\
\text { Varidol }^{\circledR}\end{array}$ & wax, pollen, honey & described \\
\hline Coumaphos & $\begin{array}{l}\text { Checkmite }^{\text {TM }} \\
\text { Perizin }^{\circledR}\end{array}$ & wax, pollen, honey & described \\
\hline Flumethrin & Bayvarol $^{\circledR}$ & wax, pollen, honey & described \\
\hline Fluvalinate & Apistan $^{\circledR}$ & wax, pollen, honey & described \\
\hline Formic acid & MAQS $^{\circledR}$ & not known & not known \\
\hline Oxalic acid & $\begin{array}{l}\text { Bienenwohl } \\
\text { Oxuvar }^{\circledR}\end{array}$ & not known & not known \\
\hline Thymol & $\begin{array}{l}\text { Apilife VAR } \\
\text { Apiguard }^{\circledR} \\
\text { Thymovar }^{\circledR}\end{array}$ & not known & not known \\
\hline
\end{tabular}

No pesticide used in honey bee medicine is $100 \%$ efficient. "Easy to use" drugs were initially seen as a simple, cheap and fast solution to honey bee disease problems, but their widespread use and loose legislation control in some countries resulted into Varroa populations rapidly developing resistance. As such, beekeepers are made reliant on more and more chemical products (11).

Following the application of these medicaments, residues can be detected throughout the beehive in products such as royal jelly but also in adult bees and brood (12). Acaricide residues constitute a significant portion of chemical contaminants of in Tables 2-7. The impacts of these chemicals on honey bee colony health are discussed systematically in the sections below.

\section{Negative Effects of Acaricides on Honey Bees}

\section{Effects on Adult Worker Bees}

A mounting body of research (reviewed in Tab. 2-7) agrees that among others, exposure of bees to some acaricides at recommended doses can lead to high bee mortality and shortened lifespan. But 
what affects the resistance of individual bees to acaricide intoxication? There seem to be several factors. Coumaphos and fluvalinate are more toxic to older bees then young bees $(15,16$ cited in 17$)$. Workers that were subjected to less stress appear to be more resistant to fluvalinate and coumaphos poisoning $(17,18)$. Bee mortality can also increase with higher outdoor temperature. Thymol is practically harmless at outdoor temperatures ranging from $5^{\circ} \mathrm{C}$ to $9^{\circ} \mathrm{C}$ (19), but was linked with high bee mortality at temperatures above $27^{\circ} \mathrm{C}(20$, 21 ). The tolerability of the oxalic acid treatment depends strongly on the method of administration used. Colonies treated with the trickling method had a significantly higher adult bee mortality then those treated with the vaporizer method (22). Quite interestingly, it has been shown that queen bees can tolerate higher doses of acaricides than workers, suggesting that the physiological differences between the two can affect pesticide sensitivity (23). Other factors that may affect the mortality of workers that contacted acaricides include their pathogen load (24), mobility (25) and the acaricide dose received $(26,27)$. On the other hand, the strength of the colony seems to not affect the resistance of bees to acaricide application (28).

Acaricide exposure also affects the bee's behaviour. Bees fed with coumaphos had reduced trophallaxis which could severely affect the food transfer and energetic distribution of the whole colony (29). Organic acaricides such as formic acid or thymol are irritant to bees and cause an increase in fanning (30 cited in $31,32,33$ ), probably to rid their hive of unwanted acaricides vapours.

Negative effects of acaricides on bees also include impaired metabolism. Bolli et al. showed that under laboratory conditions, high concentrations of formic acid in the air inhibit oxygen intake in young adult bees (34). Application of amitraz and flumethrin leads to a reduction of proteins, carbohydrates and lipids in the hemolymph of 0-, 7- and 21-day old worker bees (35). Adult bees as well as bee brood from colonies treated with amitraz (Apivar $\left.{ }^{\circledR}\right)$, flumethrin $\left(\right.$ Bayvarol $\left.^{\circledR}\right)$ and thymol (Apiguard ${ }^{\circledR}$ ) had lower levels of Glutathione S-Transferase activity (36).

Some acaricides have been shown to affect the physiology of adult worker bees. Formic acid treatment had a significant effect on the number of sensilla found in the bee's antenna. Non-significant differences were recorded for the mean length and surface area of the sensilla (37). Topically administrated oxalic acid is known to penetrate keratin and can subsequently be detected in the bee's internal organs including the digestive tract, rectum and heamolymph (38), which it can damage and cause elevated mortality (39).

Table 2: The effects of amitraz on honey bees

\begin{tabular}{|c|c|c|c|}
\hline Formulation & Exposure & Effects & Reference \\
\hline Apivar $^{\circledR}$ & $\begin{array}{l}2 \text { strips per hive, } \\
6 \text { weeks }\end{array}$ & $\begin{array}{l}\downarrow \text { proteins }, \downarrow \text { carbohydrates, } \downarrow \text { lipids in haemo- } \\
\text { lymph of adults }\end{array}$ & (31) \\
\hline Taktic $^{\circledR}$ & aerosol & associated with weakening of treated colonies & $(40)$ \\
\hline \multirow[t]{2}{*}{ in larval diet } & $\begin{array}{l}25-440 \mathrm{ppb} \text { in } \\
\text { larval diet }\end{array}$ & $\begin{array}{l}\downarrow \text { chance of pupation } \\
\downarrow \text { chance of survival to adulthood } \\
\downarrow \text { defecation }\end{array}$ & $(41)$ \\
\hline & $200 \mathrm{ppm}$ & affects expression of some proteins & $(42)$ \\
\hline $\begin{array}{l}\text { topical application } \\
\text { to adults, } 24 \text {-hour } \\
\text { exposure }\end{array}$ & $\begin{array}{l}283 \text { ppm per } \\
\text { treatment group }\end{array}$ & $\begin{array}{l}\downarrow \text { glucose dehydrogenase expression } \\
\text { may compromise cellular immunity }\end{array}$ & $(43)$ \\
\hline \multirow[t]{2}{*}{$\begin{array}{l}\text { topical application } \\
\text { to adult queen bees }\end{array}$} & $\begin{array}{l}1.0 \mu g / \mu l \text { in ac- } \\
\text { etone }\end{array}$ & $\downarrow$ brood survival & (4) \\
\hline & & $\begin{array}{l}\text { shortly after application breaks down into } 2,4 \text {-di- } \\
\text { methylaniline which is very persistent, mutagen- } \\
\text { ic, oncogenic and genotoxic }\end{array}$ & $(44,45-48)$ \\
\hline
\end{tabular}


Table 3: The effects of coumaphos on honey bees

\begin{tabular}{|c|c|c|c|}
\hline Formulation & Exposure & Effects & Reference \\
\hline \multirow{6}{*}{ Check Mite $+{ }^{\circledR}$} & \multirow{5}{*}{ two strips per colony } & $\begin{array}{l}\downarrow \text { sperm viability in drones } \\
\downarrow \text { sperm numbers in drones } \\
\downarrow \text { viability of stored sperm } \\
\text { queen bees fail to develop }\end{array}$ & $(49,50)$ \\
\hline & & $\begin{array}{l}\text { in extreme cases, acute colony positioning } \\
\text { (abnormal bee behaviour, loss of about } 2 / 3 \text { of the } \\
\text { adult population) }\end{array}$ & $(18)$ \\
\hline & & $\begin{array}{l}\uparrow \text { pkac gene (detoxification) } \\
\downarrow \text { CYP306 gene (detoxification ) } \\
\downarrow \text { VGMC gene (development) } \\
\downarrow \text { DSC37 gene (immunity) }\end{array}$ & $(51)$ \\
\hline & & alters bee gut microbiome & $(52)$ \\
\hline & & $\begin{array}{l}\uparrow \text { emergency queen cell construction } \\
\uparrow \text { adult bee mortality } \\
\downarrow \text { brood survivorship }\end{array}$ & (53) \\
\hline & & $\begin{array}{l}\downarrow \text { queen acceptance } \\
\downarrow \text { queen mating success } \\
\downarrow \text { drone production }\end{array}$ & (54) \\
\hline \multirow{4}{*}{ Perizin $^{\circledR}$} & $10 \mu \mathrm{l}$ solution & $\uparrow$ volume of haemolymph & \multirow[b]{2}{*}{$(15)$} \\
\hline & $\begin{array}{l}\text { oral administration to } \\
\text { adult worker bees }\end{array}$ & $\begin{array}{l}\text { 3-day old worker bees are three-fold less } \\
\text { susceptible to coumaphos then 8-and 13-day old } \\
\text { bees }\end{array}$ & \\
\hline & $\begin{array}{l}\text { colonies treated according } \\
\text { to manufacturer's } \\
\text { instructions }\end{array}$ & $\begin{array}{l}\uparrow \text { bee mortality if colonies were exposed } \\
\text { simultaneously to some organophosporus } \\
\text { pesticides (parathionethyl, dimethoate, dialifos) }\end{array}$ & $(55)$ \\
\hline & $20 \mathrm{ml}$ per colony & alters expression of immunoregulatory genes & $(56)$ \\
\hline \multirow{2}{*}{$\begin{array}{l}10 \% \text { coumaphos } \\
\text { strips }\end{array}$} & $\begin{array}{l}\text { one to four strips per } \\
\text { colony }\end{array}$ & $\begin{array}{l}\text { queen cells torn down } \\
\text { queen bees fail to develop }\end{array}$ & \multirow{2}{*}{$(57,58)$} \\
\hline & $1 / 4$ to $1 / 2$ strips per colony & $\begin{array}{l}\downarrow \text { queen weight } \\
\downarrow \text { ovary weight } \\
\downarrow \text { number of sperm }\end{array}$ & \\
\hline \multirow{3}{*}{$\begin{array}{l}\text { beeswax cups } \\
\text { treated with } \\
\text { coumaphos }\end{array}$} & $\begin{array}{l}1 \text { to } 100 \mathrm{mg} / \mathrm{kg} \\
\text { coumaphos in beeswax }\end{array}$ & $\downarrow$ egg laying in queens & \multirow{3}{*}{$(59,60,61)$} \\
\hline & $\begin{array}{l}10 \text { to } 100 \mathrm{mg} / \mathrm{kg} \\
\text { coumaphos in beeswax }\end{array}$ & $\begin{array}{l}\uparrow q u e e n \text { rejection } \\
\downarrow \text { queen pupal weight } \\
\downarrow \text { queen adult weight } \\
\text { skews the relationship between queen weight } \\
\text { and spermatheca size probably longer queen } \\
\text { development time }\end{array}$ & \\
\hline & $\begin{array}{l}100 \text { to } 1000 \mathrm{mg} / \mathrm{kg} \\
\text { coumaphos in beeswax }\end{array}$ & $\begin{array}{l}\uparrow \text { queen rejection } \\
\downarrow \text { queen cell acceptance } \\
\downarrow \text { queen pupal weight } \\
\downarrow \text { queen adult weight } \\
\text { queens fail to develop } \\
\text { probably longer queen development time }\end{array}$ & \\
\hline
\end{tabular}




\begin{tabular}{|c|c|c|c|}
\hline \multirow{4}{*}{$\begin{array}{l}\text { caged bees fed } \\
\text { coumaphos }\end{array}$} & $2 \mu g$ of coumaphos per cage & $\uparrow$ worker mortality & \multirow[t]{2}{*}{$(29)$} \\
\hline & $5 \mu g$ of coumaphos per cage & $\begin{array}{l}\uparrow \text { worker mortality } \\
\downarrow \text { trophallaxis }\end{array}$ & \\
\hline & $\begin{array}{l}100 \text { ppm per treatment } \\
\text { group, dissolved in sucrose } \\
\text { solution }\end{array}$ & $\begin{array}{l}\text { alters the expression of some genes related } \\
\text { to detoxification, behavioral maturation, } \\
\text { immunity }\end{array}$ & $(62)$ \\
\hline & in queen candy & $\begin{array}{l}\uparrow \text { larvae mortality } \\
\text { in combination with fluvalinate, } \downarrow \text { intestinal } \\
\text { stem cell proliferation }\end{array}$ & $(63,64)$ \\
\hline \multirow[t]{2}{*}{$\begin{array}{l}\text { coumaphos in } \\
\text { acetone }\end{array}$} & $\begin{array}{l}1-3 \mu g \text { dissolved in } 1 \mu l \text { of } \\
\text { acetone, topical application } \\
\text { to adult worker bees }\end{array}$ & $\begin{array}{l}\uparrow \text { adult worker mortality } \\
\text { toxicity of coumaphos } \uparrow \text { by up to } 3.4 \text {-fold } \\
\text { when fluvalinate is present }\end{array}$ & $(65,66)$ \\
\hline & $\begin{array}{l}5 \text { ppm coumaphos in } \\
\text { acetone, } 2 \mu l \text { topical } \\
\text { application to queens }\end{array}$ & $\begin{array}{l}\text { alters the expression of P450 subfamily } \\
\text { genes, antioxidant, immunity and } \\
\text { development genes }\end{array}$ & $(67)$ \\
\hline $\begin{array}{l}\text { acaricide-laden } \\
\text { combs from } \\
\text { a beekeeping } \\
\text { operation }\end{array}$ & $\begin{array}{l}\text { bees reared in combs } \\
\text { containing } 281 \text { to } 6311000 \\
\text { ng/g coumaphos }\end{array}$ & $\begin{array}{l}\uparrow \text { Nosema ceranae infection rate } \\
\uparrow \text { brood mortality } \\
\downarrow \text { lower longevity } \\
\text { delayed larval development } \\
\text { delayed adult emergence }\end{array}$ & $(68,69,70)$ \\
\hline in larval diet & $50 \mathrm{ppm}$ & affects expression of some proteins & (42) \\
\hline $\begin{array}{l}\text { topical application } \\
\text { to adults, 24-hour } \\
\text { exposure }\end{array}$ & $\begin{array}{l}751 \text { ppm per treatment } \\
\text { group }\end{array}$ & $\begin{array}{l}\downarrow \text { hymenoptaecin and } \downarrow \text { abaecin expression } \\
\text { may compromise cellular immunity }\end{array}$ & $(43)$ \\
\hline
\end{tabular}

Table 4: The effects of flumethrin on honey bees

\begin{tabular}{|l|l|l|l|}
\hline Formulation & Exposure & Effects & Reference \\
\hline \multirow{2}{*}{ Bayvarol $^{\circledR}$} & $\begin{array}{l}\text { 4 strips per colony for } \\
\text { six weeks }\end{array}$ & $\begin{array}{l}\downarrow \text { proteins, } \downarrow \text { carbohydrates, } \downarrow \text { lipids in } \\
\text { haemolymph of adults }\end{array}$ & (35) \\
\cline { 2 - 5 } & 12 strips per colony & $\begin{array}{l}\uparrow \text { glutathione S-transferase activity (biomarker } \\
\text { of toxic stress) in larvae, pupae and nurse bees } \\
\downarrow \text { lower protein content in treated bees }\end{array}$ & (71) \\
\hline $\begin{array}{l}\text { topical application } \\
\text { to adults }\end{array}$ & $\begin{array}{l}751 \text { ppm per treatment } \\
\text { group, 17-hour exposure }\end{array}$ & $\begin{array}{l}\uparrow \text { hymenoptaecin expression } \\
\text { may compromise cellular immunity }\end{array}$ & (43) \\
\hline
\end{tabular}

Table 5: The effects of fluvalinate on honey bees

\begin{tabular}{|l|l|l|l|}
\hline Formulation & Exposure & Effects & Reference \\
\hline \multirow{5}{*}{ Apistan $^{\circledR}$} & two strips per colony & $\begin{array}{l}\downarrow \text { drones survive the first day of their adult life } \\
\downarrow \text { drone weight } \\
\downarrow \text { drone mucus gland weight }\end{array}$ & (72) \\
\cline { 2 - 5 } & $\begin{array}{l}\text { two strips per colony } \\
\text { for 24-28 days three } \\
\text { times during the } \\
\text { season }\end{array}$ & $\begin{array}{l}\text { suspected to negatively affect queen bee } \\
\text { survivorship }\end{array}$ & (73) \\
\cline { 2 - 5 } & $\begin{array}{l}\text { two strips per colony } \\
\text { for four weeks }\end{array}$ & $\begin{array}{l}\uparrow \text { glutathione S-transferases activity } \\
\text { acetylcholinesterase activity }\end{array}$ & (74, 75) \\
\hline
\end{tabular}


Table 5: continuation

\begin{tabular}{|c|c|c|c|}
\hline Formulation & Exposure & Effects & Reference \\
\hline \multirow{4}{*}{ Apistan $^{\circledR}$} & & $\downarrow$ sperm numbers in drones & $(54)$ \\
\hline & $\begin{array}{l}\text { two strips per colony for } \\
\text { six weeks }\end{array}$ & alters bee gut microbiome & $(52)$ \\
\hline & $\begin{array}{l}\text { two strips per colony for } \\
42 \text { days }\end{array}$ & $\begin{array}{l}\uparrow \text { emergency queen cell construction } \\
\downarrow \text { brood survivorship }\end{array}$ & (53) \\
\hline & $\begin{array}{l}\text { according to } \\
\text { manufacturer's } \\
\text { instructions, for three } \\
\text { and seven days } \\
\end{array}$ & $\begin{array}{l}\text { in combination with coumaphos, } \\
\text { intestinal stem cell proliferation }\end{array}$ & $(64)$ \\
\hline $\begin{array}{l}\text { Apistan Queen } \\
\text { Tab }^{\circledR}\end{array}$ & $\begin{array}{l}\text { caged bees exposed to } \\
\text { one strip for five days }\end{array}$ & $\uparrow$ worker bee mortality & $(24)$ \\
\hline Fluwarol ${ }^{\mathrm{TM}}$ & $\begin{array}{l}\text { two strips for six } \\
\text { months }\end{array}$ & $\begin{array}{l}\text { associated with weaker colony development, } \\
\text { drone laying and queen death } \\
\text { contaminated combs probably induce } \\
\text { laying of unfertilized eggs by queens }\end{array}$ & $(76)$ \\
\hline $\begin{array}{l}10 \% \text { fluvalinate } \\
\text { strips }\end{array}$ & $\begin{array}{l}\text { eight strips per colony } \\
\text { for } 10 \text { days }\end{array}$ & $\downarrow$ queen weight & $(57,58)$ \\
\hline \multirow{2}{*}{$\begin{array}{l}1 \% \text { fluvalinate } \\
\text { strips }\end{array}$} & $\begin{array}{l}\text { caged bees exposed for } \\
3 \text { days }\end{array}$ & $\begin{array}{l}\uparrow \text { queen supersedure } \\
\uparrow \text { worker bee mortality }\end{array}$ & \multirow[t]{2}{*}{$(17)$} \\
\hline & $\begin{array}{l}\text { caged bees exposed for } \\
7 \text { days }\end{array}$ & $\uparrow$ queen mortality & \\
\hline \multirow{5}{*}{$\begin{array}{l}\text { fluvalinate in } \\
\text { acetone }\end{array}$} & $\begin{array}{l}10 \text { mg fluvalinate in } \\
\text { acetone per dish, caged } \\
\text { workers exposed for } \\
\text { 24-hours by contact } \\
\text { method }\end{array}$ & $\downarrow$ rate of odour learning & (77) \\
\hline & $\begin{array}{l}\text { dissolved in } 1-1.25 \\
\mu l \text { of acetone, topical } \\
\text { application to adult } \\
\text { workers }\end{array}$ & $\begin{array}{l}\uparrow \text { adult worker mortality } \\
\text { toxicity of fluvalinate } \uparrow \text { if other pesticides } \\
\text { are present }\end{array}$ & $(65,66)$ \\
\hline & $\begin{array}{l}0.125 \mu g-1.25 \mu g, \text { dermal } \\
\text { application }\end{array}$ & \multirow{2}{*}{$\begin{array}{l}\text { affects learning, memory and } \\
\text { responsiveness to sucrose }\end{array}$} & \multirow{2}{*}{$(78)$} \\
\hline & $\begin{array}{l}0.125 \mu g-1.25 \mu g, \text { oral } \\
\text { application }\end{array}$ & & \\
\hline & 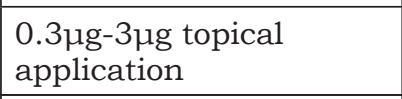 & $\downarrow$ worker mobility & $(79)$ \\
\hline $\begin{array}{l}\text { beeswax cups } \\
\text { treated with } \\
\text { fluvalinate }\end{array}$ & $\begin{array}{l}100-1000 \mathrm{mg} / \mathrm{kg} \\
\text { coumaphos in beeswax }\end{array}$ & $\begin{array}{l}\uparrow \text { queen rejection } \\
\downarrow \text { queen production } \\
\text { probably longer queen development time }\end{array}$ & $(60)$ \\
\hline \multirow[t]{2}{*}{$\begin{array}{l}\text { caged bees fed } \\
\text { fluvalinate }\end{array}$} & $\begin{array}{l}100 \mathrm{ppm} \text { per treatment } \\
\text { group, dissolved in } \\
\text { sucrose solution }\end{array}$ & $\begin{array}{l}\text { alters the expression of some genes related } \\
\text { to detoxification, behavioral maturation, } \\
\text { immunity }\end{array}$ & $(62)$ \\
\hline & in queen candy & $\uparrow$ larvae mortality & $(63)$ \\
\hline in larval diet & $100 \mathrm{ppm}$ & affects expression of some proteins & $(42)$ \\
\hline
\end{tabular}




\begin{tabular}{|l|l|l|l|}
\hline $\begin{array}{l}\text { acaricide-laden } \\
\text { combs from } \\
\begin{array}{l}\text { a beekeeping } \\
\text { operation }\end{array}\end{array}$ & $\begin{array}{l}\text { bees reared in } \\
\text { combs containing } \\
68000-104000 \\
\text { ng/g fluvalinate }\end{array}$ & $\begin{array}{l}\uparrow \text { Nosema ceranae infection rate } \\
\text { lower longevity } \\
\text { delayed larval development } \\
\text { delayed adult emergence }\end{array}$ & (68, 70) \\
\hline \multicolumn{2}{|l}{ suspected to $\uparrow$ larvae mortality } & (80) \\
\hline
\end{tabular}

Table 6: The effects of formic acid on honey bees

\begin{tabular}{|c|c|c|c|}
\hline Formulation & Exposure & Effects & Reference \\
\hline Apivarol $^{\circledR}$ & $\begin{array}{l}\text { three fumigations } \\
\text { one tablet each, } \\
\text { then } 40 \mathrm{ml} \text { of } \\
60 \% \text { formic acid } \\
\text { per colony in } \\
\text { evaporators }\end{array}$ & $\begin{array}{l}\downarrow \mathrm{pH} \text { of honey stores throughout the following } \\
\text { season }\end{array}$ & $(81)$ \\
\hline Bee Var & $\begin{array}{l}\text { one gel packet } \\
\text { per hive, two } \\
\text { treatments in two } \\
\text { week intervals }\end{array}$ & interrupts brood rearing & $(82)$ \\
\hline Miteaway $^{\mathrm{TM}}$ & $\begin{array}{l}\text { according to } \\
\text { manufacturer's } \\
\text { instructions }\end{array}$ & $\uparrow$ pkac gene (detoxification) & $(51)$ \\
\hline Varterminator $^{\mathbb{B}}$ & $\begin{array}{l}6 \% \text { formic acid gel } \\
\text { for } 20 \text { days }\end{array}$ & $\uparrow$ egg mortality by about $77 \%$ & $(83)$ \\
\hline \multirow[b]{2}{*}{$85 \%$ formic acid } & $\begin{array}{l}10 \mathrm{ml} \text { per colony } \\
\text { for } 30 \text { days }\end{array}$ & $\begin{array}{l}\uparrow \text { heat shock proteins in bee brains, a molecular } \\
\text { indicator of stress }\end{array}$ & $(84)$ \\
\hline & $\begin{array}{l}\text { in deionized } \\
\text { water, applied by } \\
\text { evaporation }\end{array}$ & cell death in gut & $(85)$ \\
\hline \multirow{6}{*}{$65 \%$ formic acid } & $\begin{array}{l}22 \mathrm{~g} \text { of formic acid } \\
\text { per absorbent pad, } \\
\text { colonies treated } \\
\text { four times at four } \\
\text { day intervals }\end{array}$ & $\begin{array}{l}\text { application can drive bees out of their hive and } \\
\text { irritate them }\end{array}$ & $(30)$ \\
\hline & $\begin{array}{l}40 \mathrm{ml} \text { per absorbent } \\
\text { pad three times in } \\
\text { weekly intervals }\end{array}$ & $\begin{array}{l}\text { application can drive bees out of their hive, } \uparrow \\
\text { fanning behaviour and irritate them }\end{array}$ & $(32)$ \\
\hline & $\begin{array}{l}200 \mathrm{ml} \text { per Ziploc } \\
\text { bag for } 40 \text { days }\end{array}$ & $\begin{array}{l}\downarrow \text { worker population } \\
\downarrow \text { worker brood area }\end{array}$ & $(86)$ \\
\hline & $\begin{array}{l}200 \mathrm{ml} \text { per } \\
\text { absorbent pad for } 6 \\
\text { weeks }\end{array}$ & $\downarrow$ brood survival & $(87)$ \\
\hline & $\begin{array}{l}\text { cotton strips } \\
\text { saturated with } \\
\text { formic acid and } \\
\text { black cumin } \\
\text { oil suspension, } \\
\text { replaced weekly for } \\
32 \text { weeks }\end{array}$ & $\begin{array}{l}\text { negative effects on sensory organs in the } \\
\text { antennae }\end{array}$ & $(37)$ \\
\hline & $\begin{array}{l}\text { one gel pack per } \\
\text { colony for } 21 \text { days }\end{array}$ & $\begin{array}{l}\downarrow \text { adult drone survival } \\
\text { delays drone production } \\
\text { drone eggs removed from combs }\end{array}$ & $(88)$ \\
\hline
\end{tabular}


Table 6: continuation

\begin{tabular}{|c|c|c|c|}
\hline Formulation & Exposure & Effects & Reference \\
\hline & 20-40ml per colony & $\downarrow$ larval feeding & \\
\hline $60 \%$ formic acid & $\begin{array}{l}200 \mathrm{ml} \text { per colony } \\
\text { for five weeks }\end{array}$ & $\begin{array}{l}\uparrow \mathrm{H}+\text { and } \mathrm{H}-\text { natural protease inhibitor activities } \\
\text { in workers } \\
\uparrow \mathrm{H}+\text { protease activities in pupae } \\
\downarrow \text { antifungal and antibacterial activities of adult } \\
\text { and larvae worker's cuticle } \\
\downarrow H+\text { protease activities in workers and larvae } \\
\downarrow \mathrm{H}+\text { and } \mathrm{H}-\text { natural protease inhibitor activities } \\
\text { in larvae and pupae } \\
\text { activates thiolic proteases }\end{array}$ & $(89)$ \\
\hline $30 \%$ formic acid & $\begin{array}{l}10 \mathrm{ml} \text { per colony } \\
\text { for } 30 \text { days }\end{array}$ & $\begin{array}{l}\uparrow \text { heat shock proteins in bee brains, a molecular } \\
\text { indicator of stress }\end{array}$ & (84) \\
\hline $\begin{array}{l}40 \mathrm{ml} \text { in } \\
\text { absorbent pad }\end{array}$ & $\begin{array}{l}\text { five applications in } \\
\text { 4-day intervals }\end{array}$ & $\begin{array}{l}\downarrow \text { area of sealed brood } \\
\text { bees clear brood cells close to the absorbent pads } \\
\text { associated with queen loss }\end{array}$ & $(90)$ \\
\hline formic acid odour & $\begin{array}{l}\text { applied by the } \\
\text { fumigation method }\end{array}$ & induces aversion in adult worker bees & $(91)$ \\
\hline \multirow{2}{*}{ fumigation } & $\begin{array}{l}60 \text { ppm formic } \\
\text { acid for } 9 \text { days per } \\
\text { overwintering room }\end{array}$ & $\begin{array}{l}\uparrow \text { worker bee mortality } \\
\text { associated with queen losses }\end{array}$ & \multirow{2}{*}{$(92)$} \\
\hline & $\begin{array}{l}45 \mathrm{ppm} \text { formic acid } \\
\text { for } 11 \text { days per } \\
\text { overwintering room }\end{array}$ & associated with queen losses & \\
\hline
\end{tabular}

Table 7: The effects of oxalic acid on honey bees

\begin{tabular}{|c|c|c|c|}
\hline Formulation & Exposure & Effects & Reference \\
\hline Api-Bioxal $^{\circledR}$ & $\begin{array}{l}35 \mathrm{~g} \text { of Api-Bioxal } \\
\text { dissolved in } 500 \mathrm{ml}\end{array}$ & $\uparrow$ adult bee mortality & (22) \\
\hline \multirow{4}{*}{$\begin{array}{l}\text { dissolved in } \\
\text { sucrose solution }\end{array}$} & $\begin{array}{l}\text { dissolved in } 50 \% \\
\text { sucrose solution, } \\
\text { applied twice by } \\
\text { trickling to hives }\end{array}$ & $\begin{array}{l}\uparrow \text { brood removal } \\
\downarrow \text { amount of brood }\end{array}$ & (93) \\
\hline & $\begin{array}{l}50 \mathrm{~mL} \text { of } 3 \% \text { oxalic } \\
\text { acid in } 32 \% \text { sucrose } \\
\text { solution }(\mathrm{w} / \mathrm{w}) \\
\text { applied once }\end{array}$ & $\uparrow$ capped brood removal & (94) \\
\hline & $\begin{array}{l}50 \mathrm{ml} \text { of } 1.5 \mathrm{~g} \text { oxalic } \\
\text { acid in sucrose } \\
\text { solution, applied by } \\
\text { trickling twice in a } \\
\text { 3-week interval }\end{array}$ & $\uparrow$ bee mortality & (39) \\
\hline & $\begin{array}{l}2 \% \text { solution of oxalic } \\
\text { acid in an aqueous } \\
50 \% \text { sucrose } \\
\text { solution by oral } \\
\text { administration }\end{array}$ & $\begin{array}{l}\text { can penetrate keratin and reach the bee's } \\
\text { internal organs }\end{array}$ & $(38)$ \\
\hline
\end{tabular}




\begin{tabular}{|c|c|c|c|}
\hline Formulation & Exposure & Effects & Reference \\
\hline \multirow{3}{*}{$\begin{array}{l}\text { dissolved in } \\
\text { sucrose solution }\end{array}$} & $\begin{array}{l}30-45 \mathrm{~g} \text { of oxalic acid per } 11 \text { of } \\
\text { sucrose solution, trickling } 5-6 \mathrm{ml} \\
\text { per bee space }\end{array}$ & $\begin{array}{l}\text { higher bee mortality with increasing } \\
\text { doses }\end{array}$ & (95) \\
\hline & $\begin{array}{l}3.2 \% \text { solution of oxalic acid } \\
\text { in } 50 \% \text { sucrose solution by } \\
\text { trickling } 5 \mathrm{ml} \text { per bee space }\end{array}$ & $\begin{array}{l}\downarrow \mathrm{pH} \text { of honey stores throughout the } \\
\text { following season } \\
\uparrow \text { heat shock proteins in bee brains, } \\
\text { a molecular indicator of stress }\end{array}$ & $(81,84)$ \\
\hline & $\begin{array}{l}4.2 \% \text { solution of oxalic acid } \\
\text { in } 50 \% \text { sucrose solution by } \\
\text { trickling } 5 \mathrm{ml} \text { per bee space }\end{array}$ & $\downarrow$ colony strength & (96) \\
\hline \multirow{6}{*}{$\begin{array}{l}\text { in aqueous } \\
\text { solution }\end{array}$} & $\begin{array}{l}\text { submersing bees in } 1 \%-2 \% \\
\text { oxalic acid in aqueous solution }\end{array}$ & $\uparrow$ adult bee mortality & \multirow{2}{*}{$(27,97)$} \\
\hline & $\begin{array}{l}\text { spraying bees with } 1 \%-1.5 \% \\
\text { oxalic acid in aqueous solution, } \\
25 \mathrm{ml} \text { per frame }\end{array}$ & $\begin{array}{l}\uparrow \text { adult bee mortality } \\
\text { colony weakening }\end{array}$ & \\
\hline & $\begin{array}{l}\text { spraying bees with } 0.56 \%- \\
2.25 \% \text { oxalic acid in aqueous } \\
\text { solution, } 50 \mathrm{ml} \text { per frame }\end{array}$ & $\begin{array}{l}\uparrow \text { colony mortality } \\
\uparrow \text { adult bee mortality } \\
\downarrow \text { brood }\end{array}$ & $(28)$ \\
\hline & $\begin{array}{l}\text { spraying bees with } 3 \% \text { oxalic } \\
\text { acid in aqueous solution, } 8 \mathrm{ml} \\
\text { per frame }\end{array}$ & $\begin{array}{l}\downarrow \text { brood } \\
\text { associated with queen death }\end{array}$ & $(98)$ \\
\hline & $\begin{array}{l}10 \%(\mathrm{w} / \mathrm{w}) \text { oxalic acid in water } \\
\text { and } 1 \% \text { Tween }{ }^{\circledR} 20,6 \mu \mathrm{L} \text { by } \\
\text { topical application per bee }\end{array}$ & $\begin{array}{l}\text { permanent damage to the bee's } \\
\text { digestive and excretory organs }\end{array}$ & (39) \\
\hline & $\begin{array}{l}10 \%-20 \% \text { oxalic acid in water, } 6 \\
\mu \mathrm{L} \text { by topical application per bee }\end{array}$ & $\begin{array}{l}\text { can penetrate keratin and reach the } \\
\text { bee's internal organs }\end{array}$ & $(38)$ \\
\hline $\begin{array}{l}\text { topical application } \\
\text { to larvae }\end{array}$ & $0.121 \mathrm{mg}$ per larva & cell death in gut and salivary glands & $(85,99)$ \\
\hline sublimation & $3.6 \mathrm{~g}$ per hive & $\begin{array}{l}\uparrow \text { heat shock proteins in bee brains, } \\
\text { a molecular indicator of stress }\end{array}$ & (84) \\
\hline
\end{tabular}

Table 8: The effects of thymol on honey bees

\begin{tabular}{|c|c|c|c|}
\hline Formulation & Exposure & Effects & Reference \\
\hline \multirow{3}{*}{ Apilife VAR ${ }^{\circledR}$} & $\begin{array}{l}\text { two wafers per colony } \\
\text { applied three times in } 51 \\
\text { days }\end{array}$ & $\downarrow$ amount of brood & $(100)$ \\
\hline & $\begin{array}{l}\text { applied for } 42 \text { days, } \\
\text { replaced at } 21 \text { days }\end{array}$ & $\begin{array}{l}\text { slows down colony development (measured } \\
\text { by adult bee population, brood, stored pollen) } \\
\text { associated with } \downarrow \text { brood }\end{array}$ & (101) \\
\hline & one tablet per colony & $\begin{array}{l}\downarrow \text { food intake in treated colonies } \\
\downarrow \text { sealed brood area } \\
\text { bees empty cells next to the tablets } \\
\text { bees remove most of the product in } 10 \text { days }\end{array}$ & $(102,103)$ \\
\hline
\end{tabular}


Table 8: continuation

\begin{tabular}{|c|c|c|c|}
\hline Formulation & Exposure & Effects & Reference \\
\hline \multirow{2}{*}{ Apilife $\mathrm{VAR}^{\circledR}$} & $\begin{array}{l}\text { one tablet per colony, } \\
\text { replaced in 10-day } \\
\text { intervals }\end{array}$ & $\begin{array}{l}\downarrow \text { sperm numbers in drones } \\
\downarrow \text { percentage of live spermatozoa in queens }\end{array}$ & $(49)$ \\
\hline & $\begin{array}{l}\text { one tablet per colony, } \\
\text { three applications in } \\
7 \text {-day intervals }\end{array}$ & $\downarrow$ phototaxis & $(104)$ \\
\hline \multirow{4}{*}{ Apiguard ${ }^{\circledR}$} & one gel per colony & $\begin{array}{l}\uparrow \text { larval and pupal mortality } \\
\downarrow \text { sealed brood area } \\
\text { bees remove most of the product in } 10 \text { days }\end{array}$ & $(103,67)$ \\
\hline & one gel per arena & $\begin{array}{l}\text { avoided by older workers } \\
\text { contact via bee antennae induces fanning } \\
\text { behaviour }\end{array}$ & $(33)$ \\
\hline & $\begin{array}{l}\text { according to } \\
\text { manufacturer's } \\
\text { instructions }\end{array}$ & $\begin{array}{l}\uparrow \text { CYP6a514 gene (detoxification) } \\
\uparrow \text { pkar gene (detoxification) } \\
\uparrow \text { pkac gene (detoxification) } \\
\downarrow \text { CYP306 gene (detoxification) } \\
\downarrow \text { VGMC gene (development) } \\
\downarrow \text { DSC37gene (immunogen) } \\
\downarrow \text { BASKgene (immunogen) }\end{array}$ & $(51)$ \\
\hline & 20 days & $\begin{array}{l}\text { when applied in conjunction with queen caging } \downarrow \\
\text { the colony population }\end{array}$ & $(105)$ \\
\hline Ecostop $^{\circledR}$ & 30 days & $\begin{array}{l}\uparrow \text { heat shock proteins in bee brains, a molecular } \\
\text { indicator of stress }\end{array}$ & $(84)$ \\
\hline Thymix & 20-40ml per colony & brood damage & $(106)$ \\
\hline Thymovar $^{\circledR}$ & & if the temperature exceeds $30^{\circ} \mathrm{C}$ brood dies off & $\begin{array}{l}\text { (Thymovar }^{\circledR} \\
\text { manufacturer } \\
\text { instructions) }\end{array}$ \\
\hline \multirow[b]{2}{*}{$\begin{array}{l}\text { thymol in } \\
\text { acetone }\end{array}$} & $\begin{array}{l}3-10 \mu \mathrm{g} \text { by topical } \\
\text { application per bee }\end{array}$ & probably $\downarrow$ drone flight activity & $(107)$ \\
\hline & $\begin{array}{l}50 \text { mg of thymol per } \\
\mathrm{kg} \text { of food by oral } \\
\text { administration to } \\
\text { larvae }\end{array}$ & altered vitellogenin expression patterns & $(108)$ \\
\hline $\begin{array}{l}\text { thymol in } \\
\text { acetone }\end{array}$ & $\begin{array}{l}500-100 \mathrm{mg} \text { of } \\
\text { thymol per kg } \\
\text { of food by oral } \\
\text { administration to } \\
\text { larvae }\end{array}$ & $\begin{array}{l}\downarrow \text { larval survival } \\
\downarrow \text { larval weight }\end{array}$ & $(108)$ \\
\hline $\begin{array}{l}\text { thymol-oil spray } \\
\text { ( } 4.8 \text { thymol } \\
\text { mol/1 in } 20 \% \\
\text { canola oil) }\end{array}$ & $\begin{array}{l}400 \mathrm{ml} \text { sprayed per } \\
\text { colony }\end{array}$ & associated with queen deaths & \multirow[t]{2}{*}{$(109)$} \\
\hline $\begin{array}{l}\text { thymol in } \\
\text { vermiculture } \\
\text { (3.6g per block) }\end{array}$ & $\begin{array}{l}\text { one block per colony } \\
\text { replaced every four } \\
\text { days for } 24 \text { days }\end{array}$ & $\uparrow$ adult bee population & \\
\hline thymol powder & $0.5 \mathrm{~g}$ per comb & application disturbed bees & $(19)$ \\
\hline
\end{tabular}




\section{Effects on Queen Bees and Drones}

In recent years, beekeepers have reported mounting problems with queen filatures. As a result, a large body of research investigated the effects of acaricides on the reproductive biology of queen bees as well as drones. In general, insecticides can lower the percentage of matings, female fecundity and reduce egg hatch in arthropods (110). In A. mellifera, where a single queen is the only reproductive individual, this could have dramatic effects. Acaricides can lower queen bee reproductive potential throughout hindering comb construction (and thus giving the queen bees less space to lay eggs), reducing the egg production, the ability to requeen and supersedure queens secessfully (111).

Several studies were devised to test whether queen bees reared in wax cups contaminated with acaricides are negatively affected. Indeed, it was shown that coumaphos treatment drastically decreases the acceptance rate of grafted queen cells (54). In addition, queen bees reared in queen cups containing $1000 \mathrm{mg} / \mathrm{kg}$ of coumaphos failed to develop. A concentration of $100 \mathrm{mg} / \mathrm{kg}$ causes over $50 \%$ queen rejection; the surviving queens weigh significantly less the untreated controls (59). Queen bees exposed to coumaphos or fluvalinate show high mortality as well as lower ovary weight, lower sperm numbers, lower body mass, physical abnormalities, atypical behaviour and other characteristics that make them unsuitable for commercial use $(49,57,58,60,61$, 112). Fluvalinate contamination of the queen cells also reduced the number of queens reared (60). Collins et al. further underlined that the adverse effects of acaricide wax contamination on queen rearing may be further deepened by stressful conditions such as excessively hot, cool, dry or humid weather, high initial $V$. destructor levels or migratory beekeeping practices (60). Under filed conditions, the pesticides beekeepers apply in their hives can combine and their synergic interactions can amplify the negative effects on honey bee reproductive characteristics. Honey bee queens raised in wax cups containing high concentrations of both coumaphos and fluvalinate, at $94 \mathrm{ppm}$ and 204 ppm respectively, had significantly lower sperm counts and sperm viability (112).

Haarmann et al. proposed two ways via which coumaphos can contaminate queen cells. Firstly, the worker bees that secrete the wax used to construct queen cells could have physically contacted coumaphos residues in the brood nest. Secondly, the developing queens may contact coumaphos via feeding by nurse bees (58). The second of the two seems less likely, since trophallaxis plays only a minor role in coumaphos distribution among bees (113). This suggests that in order to mitigate the negative impacts of acaricides on queen bees, beekeepers should frequently change old pesticide-laden combs for new uncontaminated ones.

Some acaricides have also been linked with acute mortality of queen bees. In mitefree colonies, fluvalinate application decreased queen bee survivorship by $22 \%$ after 13 months, although this difference was not statistically significant (73). According to Sokol, in colonies treated with $250 \mathrm{mg}$ strips of fluvalinate for six months, the queen ceases to lay eggs and dies. Combs contaminated with fluvalinate caused new queens to lay unfertilised eggs (76 but see 114). It has been reported that $50 \%$ of colonies treated with a thymol-oil spray as a means of Varroa control lost their queens (109). Oxalic acid and formic acid have been associated with queen losses $(90,98)$.

Less research focused on how acaricide application affects the reproductive characteristics of drones. Significantly less drones infested with Varroa mites and treated with Apistan ${ }^{\mathbb{E}}$ (fluvalinate) survive the first day of their adult life. Like this, many drones die before reaching sexual maturity. Acaricide treatment also has negative effects on drone weight, mucus glands, number of spermatozoa, sperm viability and seminal vesicles weight (50, 54, 60, 72, but see 107). Exposing drones to recommended label concentrations of coumaphos throughout their development and maturity caused a 50\% reduction in spermatozoal production (49).

Even organic treatment methods can leave negative imprints on drone reproductive fitness. Formic acid causes bees to remove their drone brood, delay their drone production and reduces drone survival (88). Likewise, it was hypothesised that thymol treatment may cause a reduction in drone flight activity (107). Acaricide treatment decreases the protein content of drone seminal fluids, probably leading to lower sperm survival (50). In effect, acaricides may cause queens to 
mate with an insufficient number of drones of a poor sperm quality. This probably results in an earlier than normal supersedure of the queen bee (115) and adversely impacts colony survivorship and productivity.

\section{Effects on Brood Development}

The widespread application of synthetic acaricides, organic acids and homemade acaricidal drugs creates a potentially deadly cocktail of chemicals that can negatively affect bee brood (116). Acaricide residues are ever-present in the hive and the brood can contact them directly in the walls of the wax combs, or can be fed dangerous doses by nurse bees (117). It has been shown that brood raised in wax combs contaminated with coumaphos and fluvalinate have higher mortality, lower longevity, delayed larval development and delayed adult emergence $(68,69)$. Laboratory studies have shown that acaricides applied at field doses can significantly increase larvae mortality (63). This could severely affect job division in the colony and make the colony less able to respond to abrupt changes in workforce demand such as in times of nectar dearth or when a large portion of the workers is lost for some reason, for example after pesticide poisoning.

Organic acaricides can be equally damaging to the brood. Apilife $\mathrm{VAR}^{\circledR}$, a thymol-based medicament, decreases brood production and causes brood removal $(100,102,103)$, especially at high in-hive temperatures (106). Thymol is in particular toxic to larvae (118). This toxicity was attributed to the strong and aromatic vapours the acaricide produces (101). Bee brood in the immediate vicinity of thymol gauze bags is readily removed by the bees (40). Gregorc et al. reported increased cell death in honey bee larvae following the application of oxalic and formic acid (85). By five days, $82 \%$ of epithelial cells of the treated larvae were affected, this was then followed by cell death in the entire larval body. Silva-Zacarin et al. further confirmed increased cell death in bee larvae treated with oxalic acid (99). A total of $18.7 \%$ of capped brood treated with oxalic acid is removed compared to $13.3 \%$ for the controls, and this difference was statistically significant (94). In addition, laboratory studies have shown that a high concentration of formic acid in the hive air (about $2500 \mathrm{ppm}$ ) halted oxygen consumption in bee brood. Subsequent in vivo studies have shown that treating bee colonies with $20-40 \mathrm{ml}$ of $60 \%$ oxalic acid reduces the rate of respiration and larval feeding (34). This may explain why colonies treated with oxalic and formic acid tend to have less brood $(86,90,93,98)$ and why bees clear cells close to absorbent pads with formic acid (90).

\section{Effect on Honey Bee Learning}

Responses to odour are key for the survival of honey bee colonies, since they play a key role in communication and foraging (111). Fluvalinate and coumaphos were found to impair bee odour learning and discriminatory abilities $(77,119)$. Whether chronic exposure to fluvalinate or coumaphos under field conditions could affect bee learning and foraging remains unknown.

\section{Effects on Disease Susceptibility}

Although acaricidal drugs are meant to alleviate parasite pressure on honey bees, they could also have the opposite effect. Short term, acaricides can efficiently control bee mites and contribute to stronger and healthier bee colonies (112). However, chronically applied sublethal doses that weaken bees could also make colonies more susceptible to other diseases.

Wu et al. (2012) found that bees that developed in combs that contained both coumaphos and fluvalinate residues subsequently suffered from higher infection with Nosema ceranae. This may be because bee brood developing in acaricideladen combs utilize more energy for detoxification purposes which results in lowered immunity $(43,51,120)$. The effects may be drastic, since nosematosis often results into colony collapse.

Previous research has shown that high levels of acaricides residue in brood combs prolong the developmental times of brood. Since bees take longer to develop, this provides more time for Varroa mites to reproduce possibly leading to a steeper population growth (69). Although no clear consensus on the effects of $A$. mellifera development time on Varroa population has been reached, it is possible that sublethal acaricide concentrations can paradoxically lead to higher mite population build up over a longer period of time.

Organic acids have been shown to negatively affect the immunity of adult worker bees. For example, formic acid was shown to impact the 
proteolytic system in the bee cuticle. Adult bees treated with formic acid had a higher $\mathrm{H}+$ protease and $\mathrm{H}$ - protease activity. The cuticle of treated bees showed lower antimicrobial activity. This may have critical implications for bee metabolism and body defence. It is widely believed that as a result of impaired metabolism and body defence, bees treated with formic acid are more susceptible to other serious diseases, namely fungal diseases (89).

Locke et al. showed that shortly after winter treatment with fluvalinate, the tires of the Deformed Wings Virus (DWV) increased for a certain period of time (121). The authors suggested that this may be because the acaricides application weakens the bees and makes them more susceptible to viral infections.

\section{Effects on Colony Strength}

The adverse sublethal effects of chemical residues on the queen, drones, adult workers and brood impair colony strength (111). Some acaricides induce aversion in worker bees, retard colony build-up and slow down food intake (91, 102, 122), leading to lower honey yields. For example, the application of thymol and formic acid causes up to $30 \%$ decrease of honey production in treated colonies, although the effect was not statistically significant $(31,90)$. Following the application of thymol or coumaphos, colonies experience a temporary period of stagnation when the amount of brood, adult honey bees and pollen does not increase or increases less than in untreated colonies $(100,101)$. These temporary stagnations and reductions in honey production may leave a lasting impact and lead to a poorer overwintering and even to colony collapses during the following winter $(76,95,97)$. Figure 1 shows how a combination of adverse side effects of acaricides could lead to elevated bee colony losses.

Past research found that the effects of acaricides vary considerably between different colonies. Firstly, the infestation rate of the colony will affects its chances of survival (86). Secondly, Rademacher and Harz pointed out that the tolerability of oxalic acid varies throughout different climates (70). While most researchers in Southern Europe find their colonies to tolerate doses of oxalic acid as high as $7 \%$ applied throughout trickling without outstanding negative effects on colony strength, researchers in Northern Europe report that their colonies tolerate much lower doses. This trend could be caused by different susceptibility to chemicals by different bee genotypes. Thirdly, the extent to which acaricide application affects colony strength is probably also dependent on the time of application (40). If the acaricides are used during periods when bees cannot fly outside of their hive, they are less able to rid themselves of residues and thus the effect may be much more serious (97). Lastly, the method of application and dose also has a decisive effect on the subsequent strength of the colony. Bee colonies treated by oxalic acid via trickling or spraying had a lower brood area then those treated by sublimation (28).

\section{Resistance to Acaricides}

As acaricides are applied routinely on an annual basis, Varroa mites are gradually building up resistance to some of them (123). Beekeepers are forced to switch to other chemicals (124, 125) which further increases the dependency on chemotherapy. It has also been suggested that by developing resistance to coumaphos, $V$. destructor may simultaneously develop resistance to other chemicals such as amitraz (124). This phenomenon is known as "cross resistance" and could mean that the apicultural industry may run out of chemicals effective against $V$. destructor in the foreseeable future.

$V$. destructor is persistently exposed to acaricide residues in bee products throughout its life. For example, Varroa mites continue to die 3 months after fluvalinate is applied. This is because fluvalinate is readily absorbed into beeswax and bee bodies and these residues remain toxic to the mites long after the application (80). Other sources claim that bee combs may show acaricidal activities even one year following the application $(126,127)$ and acaricide contamination can be detected up to seven years after the last application. As a result of this high contamination of beeswax with acaricides, Varroa mites are chronically exposed to the residues for most of their lives and probably develop resistance easier (128). If this really is the case, it is likely that thanks to acaricide residues in bee products, beekeepers are unknowingly selecting for more and more resistant mites.

The toxic effects of acaricides are not limited just to Varroa mites. Chemicals used in the hives target many other inhabitants of the hive, including 


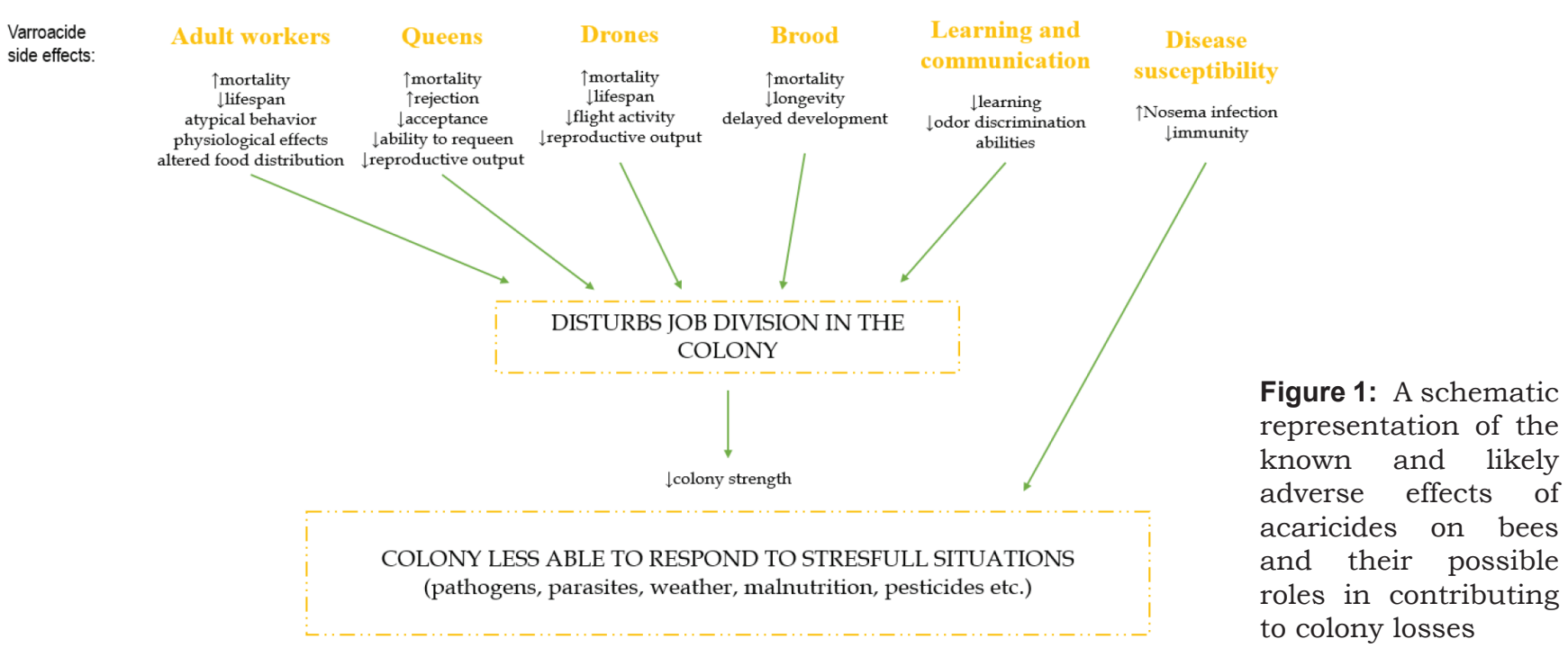

commensals and parasites. For example, a wide range of popular acaricides including fluvalinate, thymol and coumaphos exert various effects on the development of the small hive beetle (Aethina tumida) (129). This could have yet unknown impacts on the small hive beetle's populations. For example, the application of these chemicals may accelerate the beetle's development of crossresistance to other pesticides. The diverse impacts of acaricides on the development of resistance in other inhabitants of the hive will require large holistic studies in the future.

\section{Deleterious Interactions of Acaricides}

Most acaricides persist in bee products for lengthy periods of time $(125,127)$. A notable exception is amitraz, that is degraded very quickly and so does not accumulate in wax $(125,130)$. However, this does not necessarily mean that amitraz doesn't leave any residues behind. Within 3 to 4 weeks after application, amitraz breaks down into 2, 4- dimethyl phenyl formamide and 2, 4-dimethylaniline, both of which are environmentally very stable and furthermore the latter is mutagenic, oncogenic and genotoxic (44, 45-48). This shows that even readily degradable chemicals have a potential to cause significant damage to bees.

The accumulation of residues of acaricides such as coumaphos and fluvalinate can exert a lasting negative pressure on bees. Specifically, Johnson et al. demonstrated toxic interactions among the residues of acaricides as well as antimicrobial drugs and fungicides found in bee colonies (131). The toxicity of tau-fluvalinate increased when combined with other medicaments. Bees treated with coumaphos are more susceptible to poisoning by organophosporus pesticides such as parathionethyl, dimethoate, dialifos (55). Likewise, the toxicity of coumaphos increases when applied in conduction with fluvalinate (65) and bees previously treated with the antibiotic oxytetracycline were more susceptible to coumaphos and fluvalinate (132).

\section{Discussion}

Studies on the negative effects of acaricides on honey bees are relatively scarce, when compared to the total body of literature on Varroa control and many questions require further research. Beekeepers often apply acaricides at the end of the season after the last honey extraction, to minimise the risk of residues in honey. When bee colonies overwinter, which can last as long as 6 months, they are entirely dependent on the stores they produced during the year. If the medications affected bee stores, this could have a crucial effect on bee colony strength as a whole next spring. A study on humans showed that formic acid, oxalic acid and thymol added to honey significantly affected its taste (133). Could residues over sensory levels harm bees? This, among other questions, remain to be resolved with further research. So far, it has been demonstrated that formic acid induces aversion in worker bees $(91$, 122) and that formic and oxalic acids affect the $\mathrm{pH}$ 
of honey stores (81). As of present, little is known about the potential effects of pesticide residues on overwintering bees (134).

Another research topic that will require more attention in the future are the wider implications of chemotherapy for bee breeding. The heavy use of chemotherapy in apiculture may have much more pronounced effects on bee colonies then just what may seem as minute sublethal effects. Elzen et al. demonstrated that the European honey bee (A. $m$. ligustica) was significantly more tolerant to pyrethroids than African honey bees (A. m. scutellata) (135). It was suggested that European bees that are managed much more intensively, face selection pressures because of the routine application of synthetic acaricides. It thus seems that the advent chemotherapy in beekeeping significantly altered $A$. mellifera phenotype and maybe even its gene pool. This may subsequently bring serious problems in bee breeding.

Since all of the acaricides in use today have some kind of a negative side effect on bees, it is difficult to recommend the most bee-friendly product that would leave no negative health effects on colonies. The ability of beekeepers to prevent undesirable side effects of acaricides is also restrained by the fact that the manufacturing process of commercially available veterinary drugs for bees is rather non-transparent. Different formulations of the same acaricide can have very different impacts on bees. In order to overcome pest resistance problems, it is common practice now that pesticide manufacturers frequently change the formulation of their products. For example, earlier formulations of fluvalinate used in the 1980s were only slightly toxic to bees, but modern fluvalinate products are considered highly toxic (136). Furthermore, when acaricides are applied to the hive, the amount of active ingredients released may vary enormously between different commercial formulations. Factors such as nest congestion, bee activity and length of exposure may profoundly affect the amount of substance released per unit of time (137). The actual amount of active ingredient distributed through the colony may therefore be different from the amount given on the label. As such, it is hard for beekeepers and bee researchers to predict what side effect may their acaricides have. Thus, with some exaggeration it can be said that every batch of bee medicaments made has its own, unpredictable effects.
In summary, although acaricides can significantly decrease $V$. destructor infestation, they may exert negative effects if the colony is only lightly infested (86). The scale and extend of the negative impacts of acaricides is strongly influenced by factors such as the climatic conditions, the time of the year, the dose and the method of application $(26,27,28,70)$, so reaching consistent results has long been a problem. The negative effects of acaricides can be so cryptic that they can be hard to recognise by beekeepers and only cause acute poisoning if coupled with other stressors (42). Even methods used in organic beekeeping may leave long-term negative impacts on honey bee health. It is therefore suggested that acaricides are only applied as a last resort when the Varroa population reaches damaging levels. A growing number of beekeepers is turning towards zootechnical and biological methods such as drone comb removal, hyperthermia, selecting for Varroa-resistant and Varroa-tolerant bee stock, powder sugar dusting, using combs with smaller cell size and others that do not require the application of chemicals and so do not leave any residue behind and minimalize the risk of the pests developing resistance $(5,130,138,139)$. While often more time consuming and less efficient than conventional drugs, rapid developments are being made that make these methods easier to apply then before (140). Organic beekeepers are organised in a number of societies and are entitled to label their honey as "organic honey". It has been shown, that the growing interest of beekeepers in organic methods has brought forward a decrease of acaricide residues in bee products (130, 139). Novel organic Varroa control methods will certainly meet more and more popularity in the beekeeping circles in the very near future and contribute to increasing the sustainability of apiculture worldwide.

\section{References}

1. Abrol DP. Honeybee and crop pollination. In: Abrol DP. Pollination biology: biodiversity conservation and agricultural production. New York: Springer Science ; Business Media, 2012: 85-110.

2. Gallai N, Salles JM, Settele J, Vaissière BE. Economic valuation of the vulnerability of world agriculture confronted with pollinator decline. Ecol Econ 2009; 68: 810-21. 
3. Ogaba M. Household poverty reduction through bee-keeping amongst Uganda rural women. Kampala: Uganda National Apiculture Development Organisation, 2002: 5.

4. Kayode L, Lizette D, Johnson RM, Siegfried BD, Ellis MD. Effect of amitraz on queen honey bee egg and brood development. Mellifera 2014; 14: 33-40.

5. Rosenkranz P, Aumeier P, Ziegelmann B. Biology and control of Varroa destructor. J Invertebr Pathol 2010; 103: S96-119.

6. Chlebo R. Treating varroasis and monitoring bee colony losses in Slovakia. In: 8. setkání uživatelů Varroa Monitoring Systému, 10th January 2016. Brno, Czech Republic, 2016.

7. Nanetti A, Büchler R, Charriere JD, et al. Oxalic acid treatments for varroa control (review). Apiacta 2003; 38: 81-7.

8. Burgett DM. Evaluation of Apistan ${ }^{\text {тм }}$ as a control agent for Tropilaelaps clareae (Acari: Laelapidae), an Asian honey bee brood mite parasite. Am Bee J 1990; 130: 51-3.

9. Kulincevic JM, Rinderer TE, Mladjan VJ. Effects of fluvalinate and amitraz on bee lice (Braula coeca Nitzsch) in honey bee (Apis mellifera L) colonies in Yugoslavia. Apidologie 1991; 22: 43-7.

10. Vidal-Naquet N. Honeybee veterinary medicine: Apis mellifera L. Sheffield: $5 \mathrm{~m}$ Publishing, 2015: 126-7.

11. Sammataro D. Global status of honey bee mites. In: Sammataro D, Yoder JA, eds. Honey bee colony health, challenges and sustainable solutions. Boca Raton: CRC Press, 2012: 37-54.

12. Smodis Skerl MI, Kmecl V, Gregorc A. Exposure to pesticides at sublethal level and their distribution within a honey bee (Apis mellifera) colony. Bull Environ Contam Toxicol 2010; 85: 125-8.

13. Mullin CA, Frazier M, Frazier JL, Ashcraft S, Simonds R, Pettis JS. High levels of miticides and agrochemicals in North American apiaries: implications for honey bee health. PLoS One 2010; 5(3): e9754. http://journals.plos.org/plosone/article?id=10.1371/journal.pone.0009754

14. Al-Waili N, Salom K, Al-Ghamdi A, Ansari MJ. Antibiotic, pesticide, and microbial contaminants of honey: human health hazards. ScientificWorldJournal 2012; 2012: art ID 930849. https://www.hindawi.com/journals/ tswj/2012/930849/

15. van Buren NW, Mariën AG, Oudejans RC, Velthuis HH. Perizin, an acaricide to combat the mite Varroa jacobsoni: its distribution in and influence on the honeybee Apis mellifera. Physiol Entomol 1992; 17: 288-96.

16. Henderson C. Tests of chemical control agents for Varroa jacobsoni in honey-bee packages. In: Needham GR, Page Jr, RE, Definado-Baker $\mathrm{M}$, Bowman CE Africanized honey bees and mites. Chichester: Ellis Horwood, 1988: 380-6.

17. Currie RW. Fluvalinate queen tabs for use against Varroa jacobsoni Oud.: Apis mellifera L., queen and colony performance. Am Bee J 1999; 139: 871-6.

18. Gregorc A. A clinical case of honey bee intoxication after using coumaphos strips against Varroa destructor. J Apic Res 2012; 51: 142-3.

19. Chiesa F, D'agaro M. Effective control of varroatosis using powdered thymol. Apidologie 1991; 22: 135-45.

20. Gal H, Slabezki Y, Lensky Y. A preliminary report on the effect of origanum oil and thymol applications in honey bee (Apis mellifera L.) colonies in a subtropical climate on population levels of Varroa jacobsoni. Bee Sci 1992; 2: 175-80.

21. Ellis MD, Baxendale FP. Toxicity of seven monoterpenoids to tracheal mites (Acari: Tarsonemidae) and their honey bee (Hymenoptera: Apidae) hosts when applied as fumigants. J Econ Entomol 1997; 90: 1087-91.

22. Coffey MF, Breen J. The efficacy and tolerability of Api-Bioxal ${ }^{\circledR}$ as a winter varroacide in a cool temperate climate. J Apic Res 2016; 55: 65-73.

23. Dahlgren L, Johnson RM, Siegfried BD, Ellis MD. Comparative toxicity of acaricides to honey bee (Hymenoptera: Apidae) workers and queens. J Econ Entomol 2012; 105: 1895-902.

24. Pettis JS, Wilson WT, Shimanuki H, Teel PD. Fluvalinate treatment of queen and worker honey bees (Apis mellifera L.) and effects on subsequent mortality, queen acceptance and supersedure. Apidologie 1991; 22: 1-7.

25. Mautz D. Experiments on the toxicity of thymol to honeybees. Apidologie 1982; 13: 103-4.

26. Aliano NP, Ellis MD Oxalic acid: a prospective tool for reducing Varroa mite populations in package bees. Exp Appl Acarol 2009; 48: 303-9.

27. Toomemaa K, Martin AJ, Mänd M, Williams IH. Using oxalic acid in water solution in control of Varroa mites and its influence on honey bees. Agron Res 2010; 8: 345-50.

28. Al Toufailia H, Scandian L, Ratnieks FL. Towards integrated control of varroa: 2) comparing 
application methods and doses of oxalic acid on the mortality of phoretic Varroa destructor mites and their honey bee hosts. J Apic Res 2015; 54: 108-20.

29. Bevk D, Kralj J, Čokl A. Coumaphos affects food transfer between workers of honeybee Apis mellifera. Apidologie 2012; 43: 465-70.

30. Hoppe HW, Ritter W, Stephen EWC. The control of parasitic bee mites: Varroa jacobsoni, Acarapis woodi and Tropilaelaps clareae with formic acid. Am Bee J 1989; 29: 739-42.

31. Mattila HR, Otis GW. Trials of Apiguard, a thymol-based miticide. Part 1. Efficacy for control of parasitic mites and residues in honey. Am Bee J 1999; 139: 947-52.

32. Gatien P, Currie RW. Timing of acaracide treatments for control of low-level populations of Varroa destructor (Acari: Varroidae) and implications for colony performance of honey bees. Can Entomol 2003; 135: 749-63.

33. Mondet F, Goodwin M, Mercer A. Age-related changes in the behavioural response of honeybees to Apiguard ${ }^{\circledR}$, a thymol-based treatment used to control the mite Varroa destructor. J Comp Physiol A 2011; 197: 1055.

34. Bolli HK, Bogdanov S, Imdorf A, Fluri P. Action of formic acid on Varroa jacobsoni Oud. and the honeybee (Apis mellifera L.). Apidologie 1993; 24: 51-7.

35. Loucif-Ayad W, Aribi N, Smagghe G, Soltani N. A scientific note on the impact of acaracides on the nutritional biochemistry of Apis mellifera intermissa (Hymenoptera: Apidae). Apidologie 2010; 41: 135-7.

36. Loucif-Ayad W, Aribi N, Soltani N. Evaluation of secondary effects of some acaricides on Apis mellifera intermissa (Hymenoptera, Apidae): acetylcholinesterase and glutathione S-transferase activities. Eur J Sci Res 2008; 21: 642-9.

37. Zakaria ME, Allam SF. Effect of some aromatic oils and chemical acaricides on the mechanical defense behavior of honey bees against Varroa invasion and relationship with sensation responses. J Appl Sci Res 2007; 3: 653-61.

38. Nozal M, Bernal J, Gómez L, Higes M, Meana A. Determination of oxalic acid and other organic acids in honey and in some anatomic structures of bees. Apidologie 2003; 34: 181-8.

39. Martín-Hernández R, Higes M, Pérez JL, Nozal MJ, Gómez L, Meana A. Short term negative effect of oxalic acid in Apis mellifera iberiensis. Span J Agric Res 2007; 5: 474-80.
40. Marchetti S, Barbattini R, D'Agaru M. Comparative effectiveness of treatments used to control Varroa jacobsoni Oud. Apidologie 1984; 15: 363-78.

41. Toth PL. Lethal and sublethal effects of imidacloprid and amitraz on Apis mellifera Linnaeus (Hymenoptera: Apidae) larvae and pupae. Gainesville : University of Florida, 2009. Doctoral dissertation

42. Gregorc A, Evans JD, Scharf M, Ellis JD. Gene expression in honey bee (Apis mellifera) larvae exposed to pesticides and varroa mites (Varroa destructor). J Insect Physiol 2012; 58: 1042-9.

43. Garrido PM, Antúnez K, Martín M, Porrini MP, Zunino P, Eguaras MJ. Immune-related gene expression in nurse honey bees (Apis mellifera) exposed to synthetic acaricides. J Insect Physiol 2013; 59: 113-9.

44. Brimecombe R, Limson J. Voltammetric analysis of the acaricide amitraz and its degradant, 2, 4-dimethylaniline. Talanta 2007; 71: 1298-303.

45. Berry JA. Pesticides, bees and wax. Bee Culture 2009; 137: 33-5.

46. Knowles CO, Gayen AK. Penetration, metabolism and elimination of amitraz and N-(2,4-dimethylphenyl)-N-methylformamidine in Southwestern corn borer larvae (Lepidoptera: Pyralidae). J Econ Entomol 1983; 76: 410-3.

47. Knowles CO, Hamed MS. Comparative fate of amitraz and N-(2,4-dimethylphenyl)-N-methylformamidine (BTS-27271) in bollworm and tobacco budworm larvae (Lepidoptera, Noctuidae). J Econ Entomol 1989; 82: 1328-34.

48. Gregorc A, Bowen ID. Histochemical characterization of cell death in honeybee larvae midgut after treatment with Paenibacillus larvae, amitraz and oxytetracycline. Cell Biol Int 2000; 24: 319-24.

49. Burley LM. The effects of miticides on the reproductive physiology of honey bee (Apis mellifera L.) queens and drones. Blacksburg : Faculty of Virginia Polytechnic Institute, 2007. (Master of science)

50. Burley LM, Fell RD, Saacke RG. Survival of honey bee (Hymenoptera: Apidae) spermatozoa incubated at room temperature from drones exposed to miticides. J Econ Entomol 2008; 101: 1081-7.

51. Boncristiani H, Underwood R, Schwarz R, Evans JD, Pettis J. Direct effect of acaricides on pathogen loads and gene expression levels in hon- 
ey bees Apis mellifera. J Insect Physiol 2012; 58: 613-20.

52. Kakumanu ML, Reeves AM, Anderson TD, Rodrigues RR, Williams MA. Honey bee gut microbiome is altered by in-hive pesticide exposures. Front Microbiol 2016; 7: e1255. https://www.frontiersin.org/articles/10.3389/fmicb.2016.01255/ full

53. Berry JA, Hood WM, Pietravalle S, Delaplane KS. Field-level sublethal effects of approved bee hive chemicals on honey bees (Apis mellifera L). PloS One 2013; 8(10): e76536. http://journals. plos.org/plosone / article?id=10.1371/journal. pone.0076536

54. Fell RD, Tignor K. Miticide effects on the reproductive physiology of queens and drones. Am Bee J 2001; 141: 888-9.

55. Lienau FW. Effect of varroacide and pesticide treatment on honeybees. Apidologie 1990; 21: 375-7.

56. Cizelj I, Glavan G, Božič J, Oven I, Mrak V, Narat M. Prochloraz and coumaphos induce different gene expression patterns in three developmental stages of the Carniolan honey bee (Apis mellifera carnica Pollmann). Pest Biochem Physiol 2016; 128: 68-75.

57. Haarmann TK, Spivak M. The effects of fluvalinate and coumaphos in two commercial queen rearing operations. Am Bee J 2001; 141: 889.

58. Haarmann T, Spivak M, Weaver D, Weaver B, Glenn T. Effects of fluvalinate and coumaphos on queen honey bees (Hymenoptera: Apidae) in two commercial queen rearing operations. J Econ Enomol 2002; 95: 28-35.

59. Pettis J, Collins A, Wilbanks R, Feldlaufer MF. Effects of coumaphos on queen rearing in the honey bee, Apis mellifera. Apidologie 2004; 35: 605-10.

60. Collins AM, Pettis JS, Wilbanks R, Feldlaufer MF. Performance of honey bee (Apis mellifera) queens reared in beeswax cells impregnated with coumaphos. J Apic Res 2004; 43: 128-34.

61. Collins AM, Pettis JS. Correlation of queen size and spermathecal contents and effects of miticide exposure during development. Apidologie 2013; 44: 351-6.

62. Schmehl DR, Teal PE, Frazier JL, Grozinger CM. Genomic analysis of the interaction between pesticide exposure and nutrition in honey bees (Apis mellifera). J Insect Physiol 2014; 71: 177-90.

63. Zhu W, Schmehl DR, Mullin CA, Frazier
JL. Four common pesticides, their mixtures and a formulation solvent in the hive environment have high oral toxicity to honey bee larvae. PloS one 2014; 9: e77547 (11 pp). http://journals. plos.org/plosone/article?id=10.1371/journal. pone. 0077547

64. Forkpah C, Dixon LR, Fahrbach SE, Rueppell O. Xenobiotic effects on intestinal stem cell proliferation in adult honey bee (Apis mellifera L) workers. PloS One 2014; 9(3): e91180.http:// journals.plos.org/plosone / article?id=10.1371/ journal.pone.0091180

65. Johnson RM, Pollock HS, Berenbaum MR. Synergistic interactions between in-hive miticides in Apis mellifera. J Econ Entomol 2009; 102: 474-9.

66. Hillier NK, Frost EH, Shutler D.Fate of dermally applied miticides fluvalinate and amitraz within honey bee (Hymenoptera: Apidae) bodies. J Econ Entomol 2013; 106: 558-65.

67. Chaimanee V, Evans JD, Chen Y, Jackson C, Pettis JS. Sperm viability and gene expression in honey bee queens (Apis mellifera) following exposure to the neonicotinoid insecticide imidacloprid and the organophosphate acaricide coumaphos. J Insect Physopl 2016; 89: 1-8.

68. Medici SK, Castro A, Sarlo EG, Marioli JM, Eguaras MJ. The concentration effect of selected acaricides present in beeswax foundation on the survival of Apis mellifera colonies. J Apic Res 2012; 51: 164-8.

69. Wu JY, Anelli CM, Sheppard WS. Sub-lethal effects of pesticide residues in brood comb on worker honey bee (Apis mellifera) development and longevity. PloS one 2011; 6: e14720 (11 pp). http://journals.plos.org/plosone/article?id=10.1371/journal.pone.0014720

70. Rademacher E, Harz M. Oxalic acid for the control of varroasis in honey bee colonies-a review. Apidologie 2006; 37: 98-120.

71. Nielsen SA, Brødsgaard CJ, Hansen H. Effects on detoxification enzymes in different life stages of honey bees (Apis mellifera L., Hymenoptera: Apidae) treated with a synthetic pyrethroid (flumethrin). Alter Lab Anim 1999; 28: 437-43.

72. Rinderer TE, de Guzman LI, Lancaster VA, Delatte GT, Stelzer JA. Varroa in the mating yard I. The effects of Varroa jacobsoni and Apistan ${ }^{\circledR}$ on drone honey bees. Am Bee J 1999; 139: 134-9.

73. Duff SR, Furgala B. Some effects of menthol and fluvalinate on mite-free honey bee (Apis mellifera L.) colonies. Am Bee J 1992; 32: 476-7.

74. Rouibi A, Bouchema WF, Loucif-Ayad W, 
Achou M, Soltani N. Risks assessment of two acaricides (fluvalinate and oxalic acid) in Apis mellifera intermissa (Hymenoptera, Apidae): acetylcholinesterase and glutathione S-transferase activities. J Entomol Zool Stud 2016; 4: 503-8.

75. Retschnig G, Williams GR, Odemer R, et al. Effects, but no interactions, of ubiquitous pesticide and parasite stressors on honey bee (Apis mellifera) lifespan and behaviour in a colony environment. Environ Microbiol 2015; 17: 4322-31.

76. Sokol R. Effects of long-term persistence of Fluwarol (fluvalinate) on honey bee colonies. Med Wet 1996; 52: 718-20.

77. Taylor KS, Waller GD, Crowder LA. Impairment of a classical conditioned response of the honey bee (Apis mellifera L.) by sublethal doses of synthetic pyrethroid insecticides. Apidologie 1987; 18: 243-52.

78. Frost EH, Shutler D, Hillier NK. Effects of fluvalinate on honey bee learning, memory, responsiveness to sucrose, and survival. J Exp Biol 2013; 216: 2931-8.

79. Teeters BS, Johnson RM, Ellis MD, Siegfried $\mathrm{BD}$. Using video-tracking to assess sublethal effects of pesticides on honey bees (Apis mellifera L.). Environ Toxicol Chem 2012; 31: 1349-54.

80. Liu TP. Fluvalinate and its after-effects. Am Bee J 1992; 132: 398.

81. Borsuk G, Olszewski K, Paleolog J, Strachecka A, Gryzinska M. The effect of different varroacides on the acidity of winter stores and honey stores. An Univ Mariae Curie-Skłodowska Sec EE Zootech 2012; 30: 11-6.

82. Satta A, Floris I, Eguaras M, Cabras P, Garau VL, Melis M. Formic acid-based treatments for control of Varroa destructor in a Mediterranean area. J Econ Entomol 2005; 98: 267-73.

83. Giusti M, Sabelli C, Di Donato A, et al. Efficacy and safety of Varterminator, a new formic acid medicine against the varroa mite. J Apic Res 2017; 56: 162-7.

84. Gunes N, Aydın L Belenli D, Hranitz JM, Mengilig S, Selova S. Stress responses of honey bees to organic acid and essential oil treatments against varroa mites. J Apic Res 2017; 56: 175-81.

85. Gregorc A, Pogacnik A, Bowen I. Cell death in honeybee (Apis mellifera) larvae treated with oxalic or formic acid. Apidologie 2004; 35: 453-60.

86. Ostermann DJ, Currie RW. Effect of formic acid formulations on honey bee (Hymenoptera: Apidae) colonies and influence of colony and ambient conditions on formic acid concentration in the hive. J Econ Entomol 2004; 97: 1500-8.

87. Elzen PJ, Westervelt D, Lucas R. Formic acid treatment for control of Varroa destructor (Mesostigmata: Varroidae) and safety to Apis mellifera (Hymenoptera: Apidae) under southern United States conditions. J Econ Entomol 2004; 97: 1509-12.

88. de Guzman LI, Rinderer TE, Lancatser VA, Delatte GT, Stelzer A. Varroa in the mating yard. III. The effects of formic acid gel formulation on drone production. Am Bee J 1999; 139: 304-7.

89. Strachecka AJ, Paleolog J, Borsuk G, Olszewski K. The influence of formic acid on the body surface proteolytic system at different developmental stages in Apis mellifera L. workers. J Apic Res 2012; 51: 252-62.

90. Westcott LC, Winston ML. Chemical acaricides in Apis mellifera colonies: do they cause nonlethal effects? Can Entomol 1999; 131: 363-71.

91. Abramson CI. Aversive conditioning in honeybees (Apis mellifera). J Comp Psychol 1986; 100: $108-16$.

92. Underwood R., Currie R. Use of formic acid to control Varroa and tracheal mites in indoor overwintering facilities. Am Bee J 2003; 143: 323.

93. Hatjina F, Haristos L. Indirect effects of oxalic acid administered by trickling method on honey bee brood. J Apic Res 2005; 44: 172-4.

94. Gregorc A, Smodiš-Škerl MI. Toxicological and immunohistochemical testing of honeybees after oxalic acid and rotenone treatments. Apidologie 2007; 38: 296-305.

95. Charrière JD, Imdorf A. Oxalic acid treatment by trickling against Varroa destructor: recommendations for use in central Europe and under temperate climate conditions. Bee World 2002; 83: 51-60.

96. Adjlane N, Tarek EO, Haddad N. Evaluation of oxalic acid treatments against the mite Varroa destructor and secondary effects on honey bees Apis mellifera. J Arthropod Borne Dis 2016; 10: 501.

97. Toomemaa K, Martin AJ, Williams IH. The effect of different concentrations of oxalic acid in aqueous and sucrose solution on Varroa mites and honey bees. Apidologie 2010; 41: 643-53.

98. Higes M, Meana A, Suárez M, Llorente J. Negative long-term effects on bee colonies treated with oxalic acid against Varroa jacobsoni Oud. Apidologie 1999; 30: 289-92.

99. Silva-Zacarin EC, Gregorc A, de Moraes RLS. In situ localization of heat-shock proteins and cell death labelling in the salivary gland of 
acaricide-treated honeybee larvae. Apidologie 2006; 37: 507-16.

100. Ellis JD, Delaplane KS, Hood WM. Efficacy of a bottom screen device, Apistan TM, and Apilife VAR TM, in controlling Varroa destructor. Am Bee J 2001; 141: 813-6.

101. Skinner JA, Parkman JP, Studer MD. Evaluation of Apilife VAR and Checkmite+ for management of Varroa and tracheal mites in Tennessee. Am Bee J 2000; 140: 908.

102. Imdorf A, Bogdanov S, Kilchenmann V, Maquelin C. Apilife VAR: a new varroacide with thymol as the main ingredient. Bee World 1995; 76: 77-83.

103. Floris I, Satta A, Cabras P, Garau VL, Angioni A. Comparison between two thymol formulations in the control of Varroa destructor: effectiveness, persistence, and residues. J Econ Entomol 2004; 97: 187-91.

104. Alayrangues J, Hotier L, Massou I, Bertrand Y, Armengaud C. Prolonged effects of in-hive monoterpenoids on the honey bee Apis mellifera. Ecotoxicology 2016; 25: 856-62.

105. Giacomelli A, Pietropaoli M, Carvelli A, Iacoponi F, Formato G. Combination of thymol treatment (Apiguard ${ }^{\mathbb{R}}$ ) and caging the queen technique to fight Varroa destructor. Apidologie 2016; 47: 606-16.

106. Schulz S. Treatment of varroatosis with essential oils-depending on the Apilife/Var dosage. Apidologie 1993; 24: 497-9.

107. Johnson RM, Dahlgren L, Siegfried BD, Ellis MD. Effect of in-hive miticides on drone honey bee survival and sperm viability. J Apic Res 2013; 52: 88-95.

108. Charpentier G, Vidau C, Ferdy JB, Tabart $\mathrm{J}$, Vetillard A. Lethal and sub-lethal effects of thymol on honeybee (Apis mellifera) larvae reared in vitro. Pest Manag Sci 2014; 70: 140-7.

109. Whittington R, Winston ML, Melathopoulos AP, Higo HA. Evaluation of the botanical oils neem, thymol and canola sprayed to control Varroa jacobsoni (Acari: Tarsonemidae) in colonies of honey bees (Apis mellifera L., Hymenoptera: Apidae). Am Bee J 2000; 140: 567-72.

110. Bariola LA. Pink bollworms (Lepidoptera: Gelechiidae): effects of low concentrations of selected insecticides on mating and fecundity in the laboratory. J Econ Entomol 1984; 77: 1278-82.

111. Thompson HM. Behavioural effects of pesticides in bees - their potential for use in risk assessment. Ecotoxicology 2003; 12: 317-30.
112. Rangel J, Tarpy DR. The combined effects of miticides on the mating health of honey bee (Apis mellifera L.) queens. J Apic Res 2015; 54(3): 275-83.

113. Buren NWM, Mariën AGH, Velthuis HHW. The role of trophallaxis in the distribution of Perizin in a honeybee colony with regard to the control of the varroa mite. Entomol Exp Appl 1992; 65: 157-64.

114. Williams JL, Ambrose JT, Wright CG. The effect of fluvalinate (ApistanTM Queen Tabs) on queen and worker honey bees in transit and colony survivorship. Am Bee J 1994; 134: 759-62.

115. Camargo JD, Goncalves LS. Manipulation procedures in the technique of instrumental insemination of the queen honeybee Apis mellifera L. (Hymenoptera: Apidae). Apidologie 1971; 2: 239-46.

116. Woyke J. Sex determination In: Rinderer $\mathrm{TE}$, ed. Bee genetics and breeding. Hebden Bridge : Northern Bee Books, 2010: 91-119.

117. Orantes-Bermejo FJ, Pajuelo AG, Megias MM, Fernández-Pínar CT. Pesticide residues in beeswax and beebread samples collected from honey bee colonies (Apis mellifera L.) in Spain. Possible implications for bee losses. J Apic Res 2010; 49: 243-50.

118. Mattila HR, Otis GW, Daley J, Schulz T. Trials of Apiguard, a thymol-based miticide. Part 2. Non-target effects on honey bees. Am Bee J 2000; 140: 68-70.

119. Weick J, Thorn RS. Effects of acute sublethal exposure to coumaphos or diazinon on acquisition and discrimination of odor stimuli in the honey bee (Hymenoptera: Apidae). J Econ Entomol 2002; 95: 227-36.

120. Wu JY, Smart MD, Anelli CM, Sheppard WS. Honey bees (Apis mellifera) reared in brood combs containing high levels of pesticide residues exhibit increased susceptibility to Nosema (Microsporidia) infection. J Invertebr Pathol 2012; 109: 326-9.

121. Locke B, Forsgren E, Fries I, de Miranda JR. Acaricide treatment affects viral dynamics in Varroa destructor-infested honey bee colonies via both host physiology and mite control. Appl Environ Microbiol 2012; 78: 227-35.

122. Stoner A, Wilson WT, Moffett JO. Effect of long-term feeding of low doses of fenvalerate or fluvalinate in sucrose syrup on honey bees in standard-size field colonies. J Ga Entomol Soc 1984; 19: 490-8. 
123. Lodesani M, Colombo M, Spreafico M. Ineffectiveness of Apistan ${ }^{\circledR}$ treatment against the mite Varroa jacobsoni Oud. in several districts of Lombardy (Italy). Apidologie 1995; 26: 67-72.

124. Elzen JR, Baxter M, Spivak M, Wilson WT. Amitraz resistance in varroa: new discovery in North America. Am Bee J 1999; 139: 362.

125. Mayer L, Poklukar J. Coumaphos and amitraz in Slovenia honey. Apiacta 2003; 38: 54-7.

126. Moosbeckhofer R. Apistan und Bayvarol: Langzeitwirkung behandelter Waben. Bienenvater 1991; 112: 90-2.

127. Fries I, Wallner K, Rosenkranz P. Effects on Varroa jacobsoni from acaricides in beeswax. J Apic Res 1998; 37: 85-90.

128. Medici SK, Maggi MD, Sarlo EG, Ruffinengo S, Marioli JM, Eguaras MJ. The presence of synthetic acaricides in beeswax and its influence on the development of resistance in Varroa destructor. J Apic Res 2015; 54: 267-74.

129. Ellis JD, Delaplane KS. The effects of three acaricides on the developmental biology of small hive beetles (Aethina tumida). J Apic Res 2007; 46: 256-9.

130. Lodesani M, Costa C, Bigliardi M, Colombo $\mathrm{R}$. Acaricide residues in bee wax and organic beekeeping. Apiacta 2003; 38: 1-3.

131. Johnson RM, Dahlgren L, Siegfried BD, Ellis MD. Acaricide, fungicide and drug interactions in honey bees (Apis mellifera). Plos One 2013; 8(1): e54092 (10 pp). http://journals. plos.org/plosone/article?id=10.1371/journal. pone.0054092
132. Hawthorne DJ, Dively GP. Killing them with kindness? In-hive medications may inhibit xenobiotic efflux transporters and endanger honey bees. PLoS One 2011; 6(11): e26796. http:// journals.plos.org/plosone/article?id=10.1371/ journal.pone.0026796

133. Bogdanov S, Kilchenmann V, Fluri P, Bühler U, Lavanchy P. Influence of organic acids and components of essential oils on honey taste. Am Bee J 1999; 139: 61-3.

134. Boyle NK, Sheppard WS. A scientific note on seasonal levels of pesticide residues in honey bee worker tissues. Apidologie 2017; 48: 128-30.

135. Elzen PJ, Elzen GW, Rubink W. Comparative susceptibility of European and Africanized honey bee ecotypes to several insecticide classes. Southwest Entomol 2003; 28: 255-60.

136. Frazier M, Mullin C, Frazier J, Ashcraft S. What have pesticides got to do with it? Am Bee $\mathrm{J}$ 148: 521-4.

137. Wallner K. Varroacides and their residues in bee products. Apidologie 1999; 30: 235-48.

138. Bentzien C. Ökologisch imkern. Stuttgart: Franckh Kosmos, 2006.

139. Livia PO, Patrizio P, Cinzia M, Enzo M. Organic beekeeping and acaricide residues in beewax. Research in the Lazio region (Central Italy). Apiacta 2003; 38: 40-5.

140. Tihelka E. History of varroa heat treatment in Central Europe (1981-2013). Bee World 2016; 93: 4-6. 


\section{VPLIV UMETNIH IN NARAVNIH AKARICIDOV NA ZDRAVJE MEDONOSNIH ČEBEL}

\section{E. Tihelka}

Povzetek: Čebele so pomemben opraševalec kmetijskih pridelkov in gospodarsko pomemben proizvajalec izdelkov, kot sta med in vosek, ki se uporabljajo v živilski industriji, kozmetiki in medicini. Trenutno je zunanji zajedavec pršica varoja (Varroa destructor) eden največjih škodljivcev za čebele v svetu. Brez zdravljenja čebelje družine napadene z varojo večinoma propadejo v nekaj letih. Za ohranjanje nizke populacije pršic varoj čebelarji uporabljajo umetne in naravne akaricide. Najbolj priljubljeni, komercialno dostopni, so amitraz, kumafos, flumetrin, fluvalinat, mravljinčna kislina, oksalna kislina in timol. Ti akaricidi so dokaj poceni in enostavni za uporabo, vendar podaljšana uporaba povzroča hitro razvijanje odpornosti pri pršicah varoja in večjo možnost onesnaženja čebeljih pridelkov. Ostanki akaricidov so po zdravljenju lahko prisotni v visokih koncentracijah v celotnem panju in so jim čebele izpostavljene celo leto. Po uporabi je tudi satje pogosto onesnaženo z visokimi koncentracijami ostankov akaricidov, ki so jim pršice varoja kronično izpostavljene in lahko zaradi tega hitreje razvijejo odpornost. Pregledni članek povzema trenutno znanje o škodljivih učinkih konvencionalnih akaricidov na zdravje čebel. Dokazano je, da imajo številni komercialno dostopni akaricidi in njihove aktivne snovi negativne učinke na razvoj čebel, vplivajo na razmnoževalno sposobnost čebelje matice in zmanjšujejo sposobnost učenja čebel ter dolgoživost in moč kolonije. Akaricidi ne delujejo samo kot posamezne učinkovine, ampak tudi sinergistično, kar lahko dodatno slabo vpliva na zdravje čebelje družine. Nekatera zdravila lahko povzročijo znatno oslabitev čebeljih družin, lahko pajih tudi naredijo bolj dovzetne za druge bolezni, kot je nozemavost, ali bolj občutljive na slabe vremenske razmere. Vkombinaciji z drugimi stresorji lahko akaricidi prispevajo k propadu čebelje družine.

Ključne besede: umetni akaricidi; varoicidi; čebela; Apis mellifera; sinergija; ekološko čebelarjenje 